\title{
BM] open Sedentary behaviour and life expectancy in the USA: a cause-deleted life table analysis
}

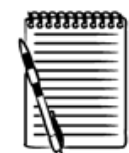

PRESS

RELEASE

To cite: Katzmarzyk PT, Lee I-M. Sedentary behaviour and life expectancy in the USA: a cause-deleted life table analysis. BMJ Open 2012;2: e000828. doi:10.1136/ bmjopen-2012-000828

- Prepublication history for this paper is available online. To view this file please visit the journal online (http://dx. doi.org/10.1136/ bmjopen-2012-000828)

Received 9 January 2012 Accepted 20 April 2012

This final article is available for use under the terms of the Creative Commons Attribution Non-Commercial 2.0 Licence; see http://bmjopen.bmj.com

${ }^{1}$ Pennington Biomedical Research Center, Louisiana State University System, Baton Rouge, Louisiana, USA ${ }^{2}$ Division of Preventive Medicine, Brigham and Women's Hospital and Harvard Medical School, Boston, Massachusetts, USA

Correspondence to Dr Peter T Katzmarzyk; peter.katzmarzyk@pbrc.edu

\section{ABSTRACT}

Objectives: To determine the impact of sitting and television viewing on life expectancy in the USA.

Design: Prevalence-based cause-deleted life table analysis.

Setting: Summary RRs of all-cause mortality associated with sitting and television viewing were obtained from a meta-analysis of available prospective cohort studies. Prevalences of sitting and television viewing were obtained from the US National Health and Nutrition Examination Survey.

Primary outcome measure: Life expectancy at birth. Results: The estimated gains in life expectancy in the US population were 2.00 years for reducing excessive sitting to $<3 \mathrm{~h} /$ day and a gain of 1.38 years from reducing excessive television viewing to $<2 \mathrm{~h} /$ day. The lower and upper limits from a sensitivity analysis that involved simultaneously varying the estimates of $R R$ (using the upper and lower bounds of the $95 \% \mathrm{Cl}$ ) and the prevalence of television viewing $( \pm 20 \%)$ were 1.39 and 2.69 years for sitting and 0.48 and 2.51 years for television viewing, respectively.

Conclusion: Reducing sedentary behaviours such as sitting and television viewing may have the potential to increase life expectancy in the USA.

Over 60 years of research on physical activity and health culminated in the release of the 2008 Physical Activity Guidelines for Americans, which recommend that adults should accumulate at least $150 \mathrm{~min}$ of moderate-tovigorous physical activity per week to attain the health benefits associated with physical activity. ${ }^{1}$ Recently, there has been considerable interest in understanding the role of sedentary behaviours on health, independent of overall physical activity levels, ${ }^{2-4}$ since one can be both sedentary and physically active (eg, an office worker who sits most of his work hours, but who also jogs regularly).

Sedentary behaviours that involve sitting for extended periods are ubiquitous in modern society. Based on self-reports, a recent survey of 20 countries documented a median of $300 \mathrm{~min} /$ day spent sitting, ranging from $\leq 180 \mathrm{~min} /$ day in Portugal,

\section{ARTICLE SUMMARY}

Article focus

- This paper presents the results of an analysis aimed at determining the effects of sedentary behaviour on life expectancy in the USA.

Key messages

- The analyses indicate that population life expectancy in the USA would be 2.00 years higher if adults reduced their time spent sitting to $<3 \mathrm{~h} /$ day and 1.38 years higher if they reduced television viewing to $<2 \mathrm{~h} /$ day.

Strengths and limitations of this study

- The use of the well-accepted prevalence-based methodology to estimate the public health burden of sedentary behaviour is a marked strength, which allows for comparability with the effects of other established risk factors.

- This study relied on self-reported engagement in sedentary behaviours rather than an objective measurement, which is a limitation.

- An important limitation of this study is the lack of adjustment for confounding beyond age and sex in the main analysis.

Brazil and Colombia to $\geq 360 \mathrm{~min} /$ day in Taiwan, Norway, Hong Kong, Saudi Arabia and Japan. ${ }^{5}$ Several studies have demonstrated positive associations between sedentary behaviours including sitting and television viewing and health outcomes such as type 2 diabetes, cardiovascular disease mortality and all-cause mortality. ${ }^{6}$ Thus, excessive time spent in sedentary behaviour is undoubtedly having an impact on public health. A recent study from Australia estimated that television viewing reduced life expectancy at birth by 1.8 years in men and 1.5 years in women. ${ }^{7}$ The purpose of this study was to determine the impact of sedentary behaviours on life expectancy in the USA.

\section{METHODS}

A prevalence-based approach was used to estimate the impact of sedentary behaviour 
on life expectancy. The population-attributable fraction (PAF) was computed from the prevalences of sedentary behaviours (defined here as sitting and television viewing) and the RR of all-cause mortality associated with these behaviours. The PAF equation used was $\sum P_{i}\left(R R_{i}-1 / R R_{i}\right)$, where $P$ is the prevalence of the risk factor among cases in stratum $\mathrm{i}(\mathrm{i}=1-2$ in this study; details are provided below). This equation produces internally valid estimates when confounding exists and adjusted RRs must be used. ${ }^{8}$

\section{RRs associated with sedentary behaviours}

Sedentary behaviour can be captured globally using questionnaires that address total daily sitting time or time spent in specific sedentary behaviours like television viewing, reading or computer use. Published studies on the associations between sitting or television viewing and all-cause mortality were identified through a systematic literature search of MEDLINE (up to end of 2011) using the following search terms: (physical inactivity OR sedentary OR television OR sitting) AND (cohort study) AND (mortality OR death). Studies that reported on the relationship between sedentary behaviours and outcomes other than all-cause mortality, or those that did not use a prospective observational cohort design, were excluded from consideration. Abstracts and papers were reviewed by both authors and any discrepancies were resolved by consensus. Studies were included if they provided RR estimates along with 95\% CIs. We pooled RR estimates from each study separately for sitting or television viewing and all-cause mortality using a random-effects meta-analysis. Pooled RR estimates were obtained for two levels of sitting and two levels of television viewing relative to a referent group in each case. In order to maintain consistency across studies, the age- and sex-adjusted RR estimates were used from each study. In cases where the authors presented only multivariable-adjusted RR estimates or used different exposure categories, we contacted them and asked them to provide this information. MIX V.2.0 software was used to conduct the meta-analysis. ${ }^{9}$

\section{Prevalence of sedentary behaviour}

The prevalences of time spent sitting and television viewing were obtained from the National Health and Nutrition Examination Survey (NHANES). The NHANES uses a complex, multistage, probability sampling design to select participants who are representative of the civilian non-institutionalised US population. ${ }^{10}$ The prevalences were obtained from the most recent NHANES data available for adults (2009-2010 for sitting; 2005-2006 for television viewing). The prevalence of sitting $(<3 \mathrm{~h}, 3-5.9 \mathrm{~h}$ and $\geq 6 \mathrm{~h} /$ day $)$ among non-pregnant adults aged 18 years and older was determined from responses to the following question in the 2009-2010 NHANES: 'How much time do you usually spend sitting on a typical day?' The prevalence of television viewing ( $<2 \mathrm{~h}, 2-3.9 \mathrm{~h}$ and $\geq 4 \mathrm{~h} /$ day) among non-pregnant adults aged 18 years and older was determined from responses to the following question in the 2005-2006 NHANES: 'Over the past 30 days, on average how many hours per day did you sit and watch TV or videos?' Data analysis followed the guidelines of the National Center for Health Statistics for analysis of NHANES data due to the complex sampling design and methods. ${ }^{11}$

The PAF equation used in this study requires the prevalence of sedentary behaviour among cases (ie, decedents) rather than from the source population (ie, NHANES prevalence). Therefore, each population prevalence obtained from the NHANES was adjusted by using the weighted average case:source prevalence ratio (ie, the prevalences among cases divided by the prevalences among the baseline source population from the prospective cohort studies).

\section{Gains in life expectancy}

The PAFs for all-cause mortality associated with sitting and television viewing were computed using the summary RR estimates obtained from the meta-analyses and the adjusted prevalences from the NHANES, as described above. Potential gains in life expectancy attributable to reducing sedentary behaviours were estimated using a cause-deleted life table analysis, which estimates years of life gained at birth if deaths from a specific cause are eliminated from the current death rates. ${ }^{12} 13$ The most current abridged life table for the USA (2009) was downloaded from WHO website. ${ }^{14}$ The PAFs computed for sedentary behaviour were used to reduce the mortality rates in the life table for adults aged 40-79 years, and the life expectancy at birth was recalculated from the new mortality rates. The difference between the current life expectancy and the causedeleted life expectancy represents the estimated gain in life expectancy from reducing the prevalence of sedentary behaviour.

\section{Sensitivity analyses}

A sensitivity analysis was used to estimate the effects of simultaneously varying the estimates of RR (using the upper and lower bounds of the $95 \% \mathrm{CI}$ ) and the prevalences of sitting and television viewing ( $\pm 20 \%)$. Furthermore, given that we relied on age- and sexadjusted RR estimates for the computation of the PAFs, we estimated the potential bias in using this approach by computing PAFs after adjusting the RR estimates for differences observed between multivariable-adjusted RRs and age- and sex-adjusted RRs in the available studies.

\section{RESULTS}

The systematic literature search yielded a total of 460 abstracts for review. From these, 455 were excluded, yielding five full articles, which were retrieved. The reasons for abstract exclusion included non-human studies $(n=4)$, reviews, commentaries or methods papers $(n=51)$ and not studying either sitting or television viewing as an exposure and all-cause mortality as the outcome $(n=400)$. All retrieved articles met the 


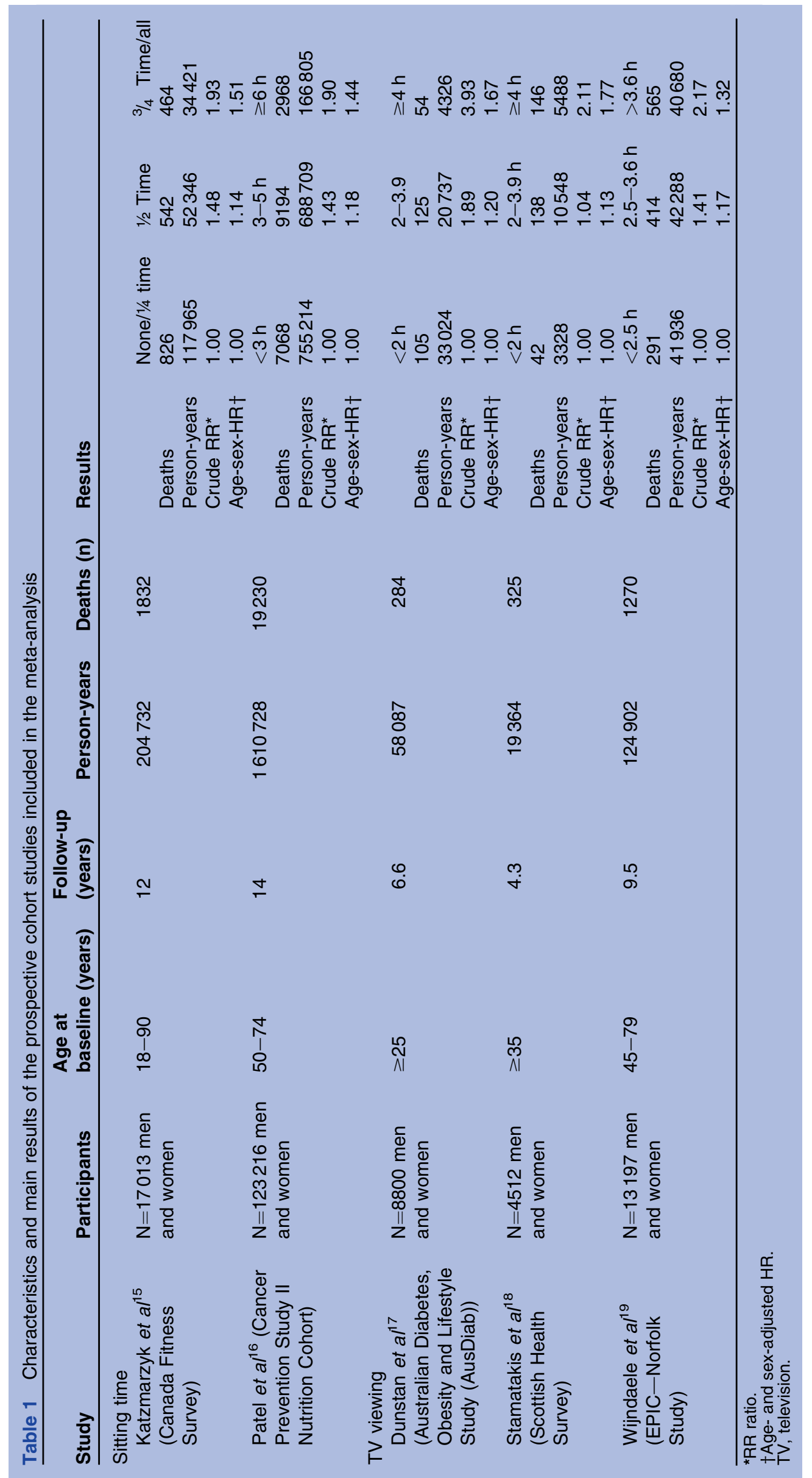


inclusion criteria and were included in the meta-analysis. The characteristics and main results of the prospective cohort studies included in the meta-analyses of sedentary behaviour and mortality are presented in table 1 . Two studies were included that investigated the association between sitting and all-cause mortality. ${ }^{15} 16$ The exposure levels of sitting in the first study were (1) none $/ 1 / 4$ of the time, (2) $1 / 2$ of the time and (3) $3 / 4$ of the time/all of the time based on activities that participants did most days of the week, ${ }^{15}$ whereas the levels in the second study were (1) $<3 \mathrm{~h}$, (2) $3-5 \mathrm{~h}$ and (3) $\geq 6 \mathrm{~h}$ of leisure-time sitting. ${ }^{16}$ Thus, three categories of exposure were used in each cohort study, and the prevalence of sitting categories from the NHANES was obtained for three groups $(<3 \mathrm{~h}, 3-5.9 \mathrm{~h}$ and $\geq 6 \mathrm{~h} /$ day $)$. However, given that the exposure categories from the Canadian cohort study were not quantifiable in terms of absolute hours per day, some misclassification may have occurred when combining the results.

Three studies were included that studied the association between television viewing and all-cause mortality. ${ }^{17-19}$ The levels of television viewing in two of the studies were (1) $<2 \mathrm{~h},(2) 2-3.9 \mathrm{~h}$ and $(3) \geq 4 \mathrm{~h},{ }^{17} 18$

\section{Weight RR $(95 \% \mathrm{Cl})$}

Level 2 versus level 1

Patel et $a^{16}$

$93.2 \% \quad 1.18$ (1.15 to 1.22$)$

Katzmarzyk et $a /^{15}$

$6.8 \% 1.14$ (1.02 to 1.27$)$

Tota

Level 3 versus level 1

$100 \% \quad 1.18$ (1.14 to 1.21$)$

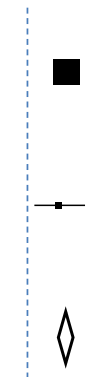

Patel et $a l^{16}$

$87.9 \% \quad 1.44$ (1.38 to 1.50$)$

Pateletaf

Katzmarzyk et $a l^{15}$

$12.1 \% \quad 1.51(1.35$ to 1.69$)$

Total

$100 \% \quad 1.45(1.39$ to 1.51$)$

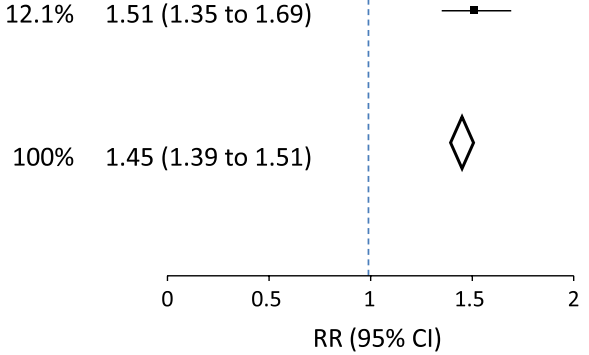

Figure 1 Risk of all-cause mortality associated with sitting. Level 3 corresponds to $3 / 4$ /all of the time for Katzmarzyk et al ${ }^{15}$ and $\geq 6 \mathrm{~h}$ for Patel et al. ${ }^{16}$ Level 2 corresponds to $1 / 2$ of the time for Katzmarzyk et al ${ }^{15}$ and 3-5 h for Patel et al. ${ }^{16}$ Level 1 corresponds to none/ $1 / 4$ of the time for Katzmarzyk et $a l^{15}$ and $<3 \mathrm{~h}$ for Patel et al. ${ }^{16}$ whereas the levels in one study were (1) $<2.5 \mathrm{~h}$, (2) 2.5-3.6 $\mathrm{h}$ and (3) $>3.6 \mathrm{~h} .{ }^{19}$ The prevalence of television viewing obtained from NHANES $(<2 \mathrm{~h}, 2-3.9 \mathrm{~h}$ and $\geq 4 \mathrm{~h}$ /day) match quite well with the exposure categories from the cohort studies.

Figure 1 presents the results of the meta-analysis for sitting and all-cause mortality. The pooled RRs were 1.18 (95\% CI 1.14 to 1.21 ) and 1.45 (95\% CI 1.39 to 1.51 ) for levels 2 and 3 versus level 1, respectively. Figure 2 presents the results for the meta-analysis for television viewing and all-cause mortality. The pooled RRs were 1.17 (95\% CI 1.04 to 1.32 ) and 1.49 (95\% CI 1.22 to 1.82) for levels 2 and 3 versus level 1, respectively.

The prevalences of sitting and television viewing in cases (decedents) and in the source population at baseline in the prospective studies are presented in table 2, along with the average case:source prevalence ratio. These ratios were applied to the population prevalences obtained from the NHANES in order to estimate the prevalences among cases in the population, which are required for inclusion in the PAF calculations. Figure 3 presents the weighted population prevalences of sitting and television viewing in the NHANES, along with the

\section{Weight RR (95\% Cl)}

Level 2 versus level 1

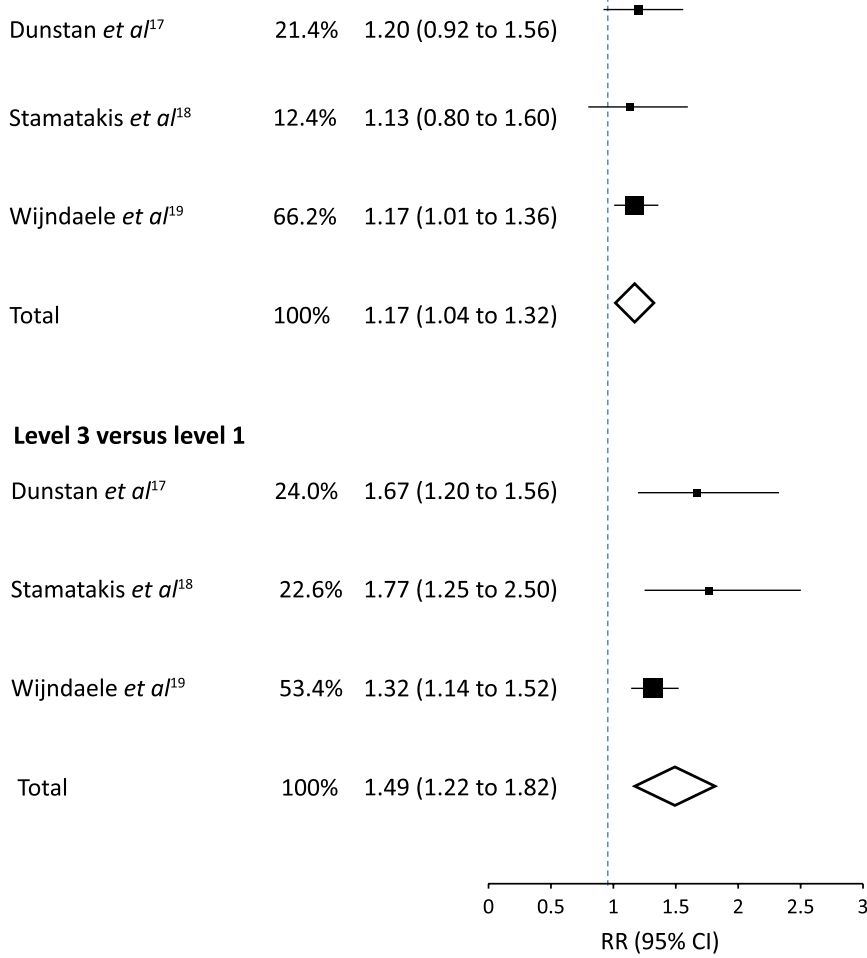

Figure 2 Risk of all-cause mortality associated with television viewing. Level 3 corresponds to $\geq 4 \mathrm{~h}$ for Dunstan et $\mathrm{al}{ }^{17}$ and Stamatakis et al ${ }^{18}$ and $>3.6 \mathrm{~h}$ for Wijndaele et al. ${ }^{19}$ Level 2 corresponds to $2-3.9 \mathrm{~h}$ for Dunstan et a/ ${ }^{17}$ and Stamatakis et $a l^{18}$ and 2.5-3.6 $\mathrm{h}$ for Wijndaele et al. ${ }^{19}$ Level 1 corresponds to $<2 \mathrm{~h}$ for Dunstan et $a l^{17}$ and Stamatakis et $a l^{18}$ and $<2.4 \mathrm{~h}$ for Wijndaele et al. $^{19}$ 
Table 2 Prevalences of sitting and TV viewing at baseline in the prospective cohort studies included in the meta-analysis

\begin{tabular}{|c|c|c|c|c|}
\hline \multirow{2}{*}{$\frac{\text { Study }}{\text { Sitting time }}$} & \multicolumn{4}{|l|}{ Prevalences } \\
\hline & \multicolumn{4}{|c|}{ Sitting time } \\
\hline Katzmarzyk et al ${ }^{15}$ & & None $/ 1 / 4$ time & $1 / 2$ Time & $3 / 4$ Time/all \\
\hline \multirow[t]{3}{*}{ (Canada Fitness Survey) } & Source prevalence $(\%)^{*}$ & 56.9 & 25.7 & 17.4 \\
\hline & Case prevalence $(\%) \dagger$ & 45.1 & 29.6 & 25.3 \\
\hline & Case:source ratio & 0.79 & 1.15 & 1.45 \\
\hline Patel et al ${ }^{16}$ (Cancer Prevention & & $<3 \mathrm{~h}$ & $3-5 h$ & $\geq 6 \mathrm{~h}$ \\
\hline \multirow[t]{4}{*}{ Study II Nutrition Cohort) } & Source prevalence $(\%)^{\star}$ & 46.2 & 43.1 & 10.7 \\
\hline & Case prevalence $(\%) \dagger$ & 36.8 & 47.8 & 15.4 \\
\hline & Case:source ratio & 0.80 & 1.11 & 1.44 \\
\hline & Average case:source ratio $\neq$ & 0.80 & 1.11 & 1.44 \\
\hline \multicolumn{5}{|l|}{ TV viewing } \\
\hline Dunstan et al ${ }^{17}$ (Australian Diabetes, & & $<2 \mathrm{~h}$ & $2-3.9 h$ & $\geq 4 \mathrm{~h}$ \\
\hline \multirow[t]{3}{*}{ Obesity and Lifestyle Study (AusDiab)) } & Source prevalence $(\%)^{*}$ & 56.5 & 35.9 & 7.6 \\
\hline & Case prevalence $(\%) \dagger$ & 37.0 & 44.0 & 19.0 \\
\hline & Case:source ratio & 0.65 & 1.23 & 2.50 \\
\hline \multirow[t]{4}{*}{ Stamatakis et al $^{18}$ (Scottish Health Survey) } & & $<2 \mathrm{~h}$ & $2-3.9 h$ & $\geq 4 \mathrm{~h}$ \\
\hline & Source prevalence $(\%)^{\star}$ & 17.1 & 54.1 & 28.8 \\
\hline & Case prevalence $(\%) \dagger$ & 13.4 & 45.2 & 41.4 \\
\hline & Case:source ratio & 0.78 & 0.84 & 1.44 \\
\hline \multirow[t]{5}{*}{ Wijndaele et $\mathrm{al}^{19}$ (EPIC-Norfolk Study) } & & $<2.5 \mathrm{~h}$ & $2.5-3.6 \mathrm{~h}$ & $>3.6 \mathrm{~h}$ \\
\hline & Source prevalence $(\%)^{*}$ & 33.3 & 33.8 & 32.9 \\
\hline & Case prevalence $(\%) \dagger$ & 22.9 & 32.6 & 44.5 \\
\hline & Case:source ratio & 0.69 & 0.96 & 1.35 \\
\hline & Average case:source ratio $\ddagger$ & 0.69 & 1.03 & 1.75 \\
\hline
\end{tabular}

prevalences after adjustment for the average case:source prevalence ratios from table 2.

The PAFs for all-cause mortality associated with sitting and television viewing were $27 \%$ and $19 \%$, respectively. The results of the life table analyses indicate a gain in life expectancy from reducing the prevalence of sedentary behaviour from level 2 or 3 to level 1 results in a gain of 2.00 years for reducing sitting prevalence and a gain of 1.38 years from reducing television viewing prevalence. The lower and upper limits from the sensitivity analyses were 1.39 and 2.69 years for sitting and 0.48 and 2.51 years for television viewing, respectively.

Data from two studies of television viewing ${ }^{17} 18$ and one study of sitting ${ }^{15}$ were available, where both age- and sex-adjusted as well as multivariable-adjusted RR estimates for all-cause mortality were presented. For sitting, the RR for level 2 versus level 1 was the same and the RR for level 3 versus level 1 was $6 \%$ lower for the multivariable compared with the age- and sex-adjusted RR. For television viewing, the RR for level 2 versus level 1 was $9.6 \%$ lower, and for level 3 versus level 1, the RR was $16.4 \%$ lower for the multivariable compared with the age- and sex-adjusted RR. After reducing the RR estimates obtained from the meta-analysis by these percentages, the estimates of attributable life expectancy were 1.76 years for sitting and 0.93 years for television viewing.

\section{DISCUSSION}

The results of this study indicate that limiting sitting to $<3 \mathrm{~h} /$ day and limiting television viewing to $<2 \mathrm{~h} /$ day may increase life expectancy at birth in the USA by approximately 2.0 and 1.4 years, respectively, assuming a causal relationship. The PAF provides a theoretical estimate of the effects of a risk factor on an outcome at the population level, in this case, all-cause mortality. The results indicate that sedentary behaviours are accounting for between 1.4 and 2.0 years of life expectancy at birth. This should not be interpreted to mean that people who are more sedentary can expect to live 1.4 or 2.0 years less than someone who does not engage in these behaviours as much. Life expectancy is a population statistic and it does not apply to individuals. A recent meta-analysis of television viewing and all-cause mortality estimated that the RR of all-cause mortality was 1.13 (95\% CI 1.07 to $1.18) / 2 \mathrm{~h}$ of daily television viewing, which corresponded with 104 deaths per 100000 people in the USA. $^{4}$

This study has several strengths and limitations that warrant discussion. The use of the well-accepted prevalence-based PAF methodology to estimate the public health burden of sedentary behaviour is a marked strength, which allows for comparability with the effects of other established risk factors. However, the PAF provides a theoretical estimate of the effects of a risk factor on a health outcome, and further research is required 

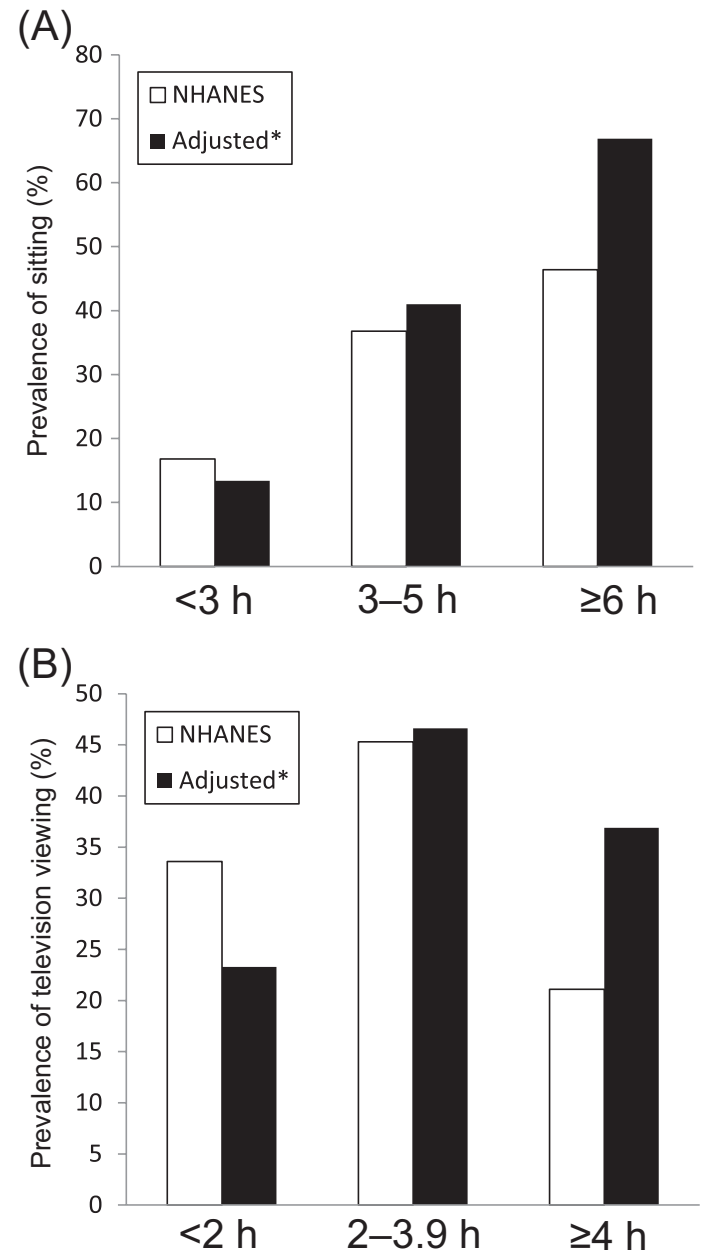

Figure 3 Prevalences of $(A)$ sitting and $(B)$ television viewing in the US National Health and Nutrition Examination Survey (NHANES). *Adjusted prevalences of sitting and television viewing using the weighted average case:source prevalence ratio obtained from cohort studies of sedentary behaviour and all-cause mortality.

using an incidence-based approach. Our analysis assumes that there is a causal relationship between sedentary behaviour and mortality. While studies using randomised designs are not possible, further observational studies, which control for confounding, will add to the evidence for causation. Another major strength of this study is the use of representative population data from the NHANES to quantify the exposure of the population to sedentary behaviours. However, our analysis estimated the overall gains in life expectancy at the population level and assumes that the effects of sedentary time on all-cause mortality are consistent across age and demographic subgroups of the population. Each of the cohort studies provided multivariable-adjusted RR estimates for sedentary behaviour and mortality using different combinations of covariates, and we chose to use summary RR estimates based on RR adjusted for age and sex in order to maintain consistency across studies. The degree to which this approach has yielded an overestimation of the independent effect of sedentary behaviour on life expectancy is not known, as important confounders associated with both sedentary behaviour and premature mortality may have been unmeasured or inadequately adjusted for in the primary studies, and we have not made further adjustments for confounding in our analyses. We conducted a sensitivity analysis by reducing the $\mathrm{RR}$ from the meta-analysis by the amount observed between age- and sex-adjusted versus multivariable RR estimates from three studies, ${ }^{15} 1718$ and the estimates of attributable life expectancy were reduced to 1.76 years for sitting and to 0.93 years for television viewing.

This study relied on self-reported engagement in sedentary behaviours, which introduces the possibility for error and recall bias. Future cohort studies should attempt to better quantify sedentary behaviour using objective activity monitors; however, the self-reported estimates of sitting reported in the NHANES are similar to those obtained for the USA in a study of 20 countries, ${ }^{5}$ which indicates some face validity to the results. For television viewing, the categories of exposure reported in two of the cohort studies $(<2,2-3.9$ and $\geq 4 \mathrm{~h})$ matched the categories reported in the NHANES; however, Wijndaele et $a l^{19}$ used a lower threshold for the upper category $(>3.6 \mathrm{~h})$, which may have resulted in an underestimate of the effects of television viewing on life expectancy. The studies on sitting used different exposure categories (ie, total sitting time versus leisure-time sitting only), so assumptions had to be made when estimating the exposure levels in the NHANES. Inaccuracies associated with the assessment of sedentary behaviour using self-report methods in the cohort studies would have led to regression dilution bias and resulted in underestimates of the association with all-cause mortality.

The results of several recent studies have suggested that the effects of sedentary behaviour on health may be independent of the effects of physical activity per se. ${ }^{2} 20$ It has been estimated that a lack of leisure-time physical activity accounts for approximately 0.9 years of life expectancy at birth in Canada. ${ }^{21}$ Given that the studies used to derive the summary RR estimates for the current study in many cases included leisure-time physical activity as a covariate in multivariable-adjusted models, and this did not appreciably change the estimates of RR for sedentary behaviour, the estimates of the effects on life expectancy may also be considered independent. Current life expectancy in the USA (2009) is 78.5 years. ${ }^{14}$ The effects of sedentary behaviour on life expectancy reported in this study are on a similar order of magnitude as other chronic disease risk factors. For example, it has been estimated that obesity accounts for between 0.30 and 1.08 years of population life expectancy at birth in the USA, depending on gender, ethnicity and severity of obesity. ${ }^{22}$ A more recent study has estimated that the current distribution of body mass index, compared with an optimal distribution (a mean of $\left.21 \mathrm{~kg} / \mathrm{m}^{2}\right)$, accounts for 1.3 years of current life 
expectancy at birth in both men and women in the USA. $^{23}$ Results from the same study indicate that smoking is also associated with 2.5 and 1.8 years of life expectancy at birth in men and women, respectively. ${ }^{23}$

Sitting time is a global measure of sedentary behaviour, whereas television viewing is somewhat more specific. A recent review found that domain-specific behaviours such as television viewing are recalled with more reliability than global measures of sitting and sedentary behaviour. ${ }^{24}$ The degree to which differences in reliability between the measures used in this study may have affected the estimates is not known. There is some evidence to suggest that using a single global question to measure sitting produces lower estimates than more detailed domain-specific questions. ${ }^{24} 25$ Thus, it is likely that the prevalences of higher levels of sitting reported in this study from the NHANES are likely conservative. Using objective monitoring (accelerometry) in the 2003-2004 NHANES, Matthews et $a l^{26}$ reported that US adults spend approximately $7.7 \mathrm{~h} /$ day engaged in sedentary behaviour. There are several potential mechanisms that could explain the association between sedentary behaviour and all-cause mortality rates. Sedentary behaviour is associated with an increased risk of the development of chronic conditions such type 2 diabetes and cardiovascular disease. ${ }^{6}$ Furthermore, human and animal studies indicate that sedentary behaviour is associated with elevated cardiometabolic biomarkers and a poor risk factor profile. ${ }^{27} 28$ For example, hindlimb suspension (unloading) in rats results in marked immediate decreases in lipoprotein lipase activity, triglyceride uptake into red skeletal muscle and reductions in the concentration of highdensity lipoprotein cholesterol. ${ }^{29}$ Future intervention research is required to determine the causal pathways between sedentary behaviour and health outcomes that have the potential to impact mortality rates.

In conclusion, the results of this study indicate that extended sitting time and television viewing may have the potential to reduce life expectancy in the USA. Given that the results from objective monitoring of sedentary time in the NHANES has indicated that adults spend an average of $55 \%$ of their day engaged in sedentary pursuits, ${ }^{26}$ a significant shift in behaviour change at the population level is required to make demonstrable improvements in life expectancy. Further research using intervention designs is required to determine the effects of reducing sedentary behaviour on health outcomes and to make recommendations regarding the safe levels of sedentary behaviour for the population.

Contributors Both authors were responsible for study conception and design. PTK was responsible for data analysis. Both authors drafted and critically revised the manuscript and approved the final version to be published. PTK is the guarantor for the study.

Funding This research received no specific grant from any funding agency in the public, commercial or non-profit sectors.

Competing interests None.
Provenance and peer review Not commissioned; externally peer reviewed.

Data sharing statement There are no additional data available.

\section{REFERENCES}

1. US Department of Health and Human Services. 2008 Physical Activity Guidelines for Americans. US Department of Health and Human Services. www.health.gov/paguidelines (accessed 10 May 2012).

2. Katzmarzyk PT. Physical activity, sedentary behavior, and health: paradigm paralysis or paradigm shift? Diabetes 2010;59:2717-25.

3. Thorp AA, Owen N, Neuhaus M, et al. Sedentary behaviors and subsequent health outcomes in adults a systematic review of longitudinal studies, 1996-2011. Am J Prev Med 2011;41:207-15.

4. Grontved A, Hu FB. Television viewing and risk of type 2 diabetes, cardiovascular disease, and all-cause mortality: a meta-analysis. JAMA 2011;305:2448-55.

5. Bauman A, Ainsworth BE, Sallis JF, et al. The descriptive epidemiology of sitting: a 20-country comparison using the International Physical Activity Questionnaire (IPAQ). Am J Prev Med 2011;41:228-35.

6. Proper KI, Singh AS, van Mechelen W, et al. Sedentary behaviors and health outcomes among adults: a systematic review of prospective studies. Am J Prev Med 2011;40:174-82.

7. Veerman JL, Healy GN, Cobiac LJ, et al. Television viewing time and reduced life expectancy: a life table analysis. $\mathrm{Br} J$ Sports Med. Published Online First: 15 August 2011. doi:10.1136/ bjsm.2011.085662

8. Rockhill B, Newman B, Weinberg C. Use and misuse of population attributable fractions. Am J Public Health 1998;88:15-19.

9. Bax L. Professional software for meta-analysis in Excel. Version 2.01.4. BostatXL. 2011. http://www.meta-analysis-made-easy.com

10. Centers for Disease Control and Prevention (CDC). National Health and Nutrition Examination Survey. 2011. http://www.cdc.gov/nchs/ nhanes.htm (accessed 7 Oct 2011).

11. Centers for Disease Control and Prevention (CDC), National Center for Health Statistics. The National Health and Nutrition Examination Survey Analytic and Reporting Guidelines. 2006. http://www.cdc.gov/ nchs/nhanes/nhanes2003-2004/analytical_guidelines.htm (accessed 7 Oct 2011)

12. Manuel DG, Luo W, Ugnat AM, et al. Cause-deleted health-adjusted life expectancy of Canadians with selected chronic conditions. Chronic Dis Can 2003;24:108-15.

13. Newman SC. Formulae for cause-deleted life tables. Stat Med 1987;6:527-8.

14. World Health Organization. Global Health Observatory Data Repository. 2011. http://apps.who.int/ghodata/ (accessed 7 Oct 2011).

15. Katzmarzyk PT, Church TS, Craig CL, et al. Sitting time and mortality from all causes, cardiovascular disease, and cancer. Med Sci Sports Exerc 2009;41:998-1005.

16. Patel AV, Bernstein L, Deka A, et al. Leisure time spent sitting in relation to total mortality in a prospective cohort of US adults. $A m \mathrm{~J}$ Epidemiol 2010;172:419-29.

17. Dunstan DW, Barr EL, Healy GN, et al. Television viewing time and mortality: the Australian Diabetes, Obesity and Lifestyle Study (AusDiab). Circulation 2010;121:384-91.

18. Stamatakis E, Hamer M, Dunstan DW. Screen-based entertainment time, all-cause mortality, and cardiovascular events population-based study with ongoing mortality and hospital events follow-up. J Am Coll Cardiol 2011;57:292-9.

19. Wijndaele $\mathrm{K}$, Brage $\mathrm{S}$, Besson $\mathrm{H}$, et al. Television viewing time independently predicts all-cause and cardiovascular mortality: the EPIC Norfolk Study. Int J Epidemiol 2011;40:150-9.

20. Tremblay MS, Colley RC, Saunders TJ, et al. Physiological and health implications of a sedentary lifestyle. Appl Physiol Nutr Metab 2010;35:725-40.

21. Katzmarzyk PT. Physical inactivity and life expectancy in Canada. $J$ Phys Act Health 2006;3:381-9.

22. Olshansky SJ, Passaro DJ, Hershow RC, et al. A potential decline in life expectancy in the United States in the 21st century. N Engl J Med 2005;352:1138-45.

23. Danaei G, Rimm EB, Oza S, et al. The promise of prevention: the effects of four preventable risk factors on national life expectancy and life expectancy disparities by race and county in the United States. PLoS Med 2010;7:e1000248.

24. Healy GN, Clark BK, Winkler EA, et al. Measurement of adults' sedentary time in population-based studies. Am J Prev Med 2011;41:216-27.

25. Clemes SA, David BM, Zhao Y, et al. Validity of two self-report measures of sitting time. J Phys Act Health. Published Online First: 12 May 2011 
26. Matthews CE, Chen KY, Freedson PS, et al. Amount of time spent in sedentary behaviors in the United States, 2003-2004. Am J Epidemiol 2008;167:875-81.

27. Healy GN, Matthews CE, Dunstan DW, et al. Sedentary time and cardio-metabolic biomarkers in US adults: NHANES 2003-06. Eur Heart J 2011;32:590-7.
28. Hamilton MT, Hamilton DG, Zderic TW. Exercise physiology versus inactivity physiology: an essential concept for understanding lipoprotein lipase regulation. Exerc Sport Sci Rev 2004;32:161-6.

29. Bey L, Hamilton MT. Suppression of skeletal muscle lipoprotein lipase activity during physical inactivity: a molecular reason to maintain daily low-intensity activity. J Physiol 2003;551:673-82. 